\title{
Comparison of Time Concept Modeling for Querying Temporal Information in OWL and RDF
}

\author{
Bahareh Bahadorani \\ Department of Computer Engineering, Isfahan (Khorasgan) branch, Isfahan, Azad University, Isfahan, IRAN \\ E-mail: bahareh.bahadorani@yahoo.com \\ Ahmad Zaeri \\ Department of Software Engineering, Faculty of Computer Engineering, University of Isfahan, IRAN \\ E-mail: zaeri@eng.ui.ac.ir
}

\begin{abstract}
Ontology is an important factor in the integration of heterogeneous semantic information. Description logic, as a formal language for expressing ontologies, does not include the necessary features to create a temporal dimension in the relationships among concepts. It is critical to introduce time concepts to model temporal data and relate them to other non-temporal data recorded in ontology. Current query languages in the semantic web are not able to respond to temporal questions; thus, another important issue is to have the appropriate methods for answering temporal questions. In this paper, temporal modeling methods in OWL and RDF are assessed and the temporal query languages for expressing queries in the semantic web are categorized and compared.
\end{abstract}

Index Terms-Ontology, temporal model, description logic, temporal query language.

\section{INTRODUCTION}

Ontologies, allow sharing, integrating and managing data in the semantic web. The concept of time is inherently linked to many other scientific concepts, because events occur during time intervals. It is important to consider the concept and determine how it can be captured in databases. Therefore, in addition to being stored as a concept in the knowledge base, its semantic relationships with other concepts need to be defined. To this end, several models have been introduced, wherein time is assessed based on the authors' viewpoint.

In recent years, the Semantic Web Community has developed a number of tools and applications such as reasoners, editors, and querying systems in order to work with temporal data [22], [24]. Despite having numerous advantages and overcoming the shortcomings of the previous models, in most cases, ontologies and ontology query languages cannot fully express the temporal knowledge needed in many applications.

Quite a number of papers, particularly [19], highlight the importance of time in the semantic web by fully expressing most temporal concepts [2], [6], [17-19],[2425], [31], [34], [35], [42]. Based on these papers, it is clear that a gap exists in describing temporal query languages. Furthermore, due to the variety of these languages, users may be forced to spend large amounts of time for comparing the languages before one can be chosen.

This paper aims to compare various temporal modeling methods, in particular TOQL and SQWRL as most frequently used temporal query languages in the semantic web.

The remainder of this paper is organized as follows. Related works are presented in Sec 2, which deals with time and proposed temporal models as well as implemented several systems. Temporal query languages for the semantic web are discussed in Sec 3 and followed by concluding remarks in Sec 4 .

\section{RELATED WORKS}

Currently, the concept of time is not incorporated into many semantic web tools and technologies used to define relations in OWL. Therefore, the most important step is to identify models which are capable of introducing time into the related concepts. These models can be divided into two categories.

The first category consists of methods wherein the basic ontology, without temporal concepts, is obtained from the user. Temporal concepts, classes, and relations are then added to the base ontology. In this approach, the user does not need to be familiar with high-level concepts and the created relations. This can be done by the tab plugin added to the Protégé editor. Both [8] and [42] adopt the first category.

In the second category, while designing the class model in the analysis and design stage of the ontology concepts, the user creates the time-related classes. In other words, the ontology is created along with all the temporal and non-temporal concepts.

Most large ontologies follow the second approach, whereas smaller ontologies tend to take the first approach. 
Important theories such as Horn-clause Temporal Logic, Allen's Interval Algebra [5] and Davidson's Event Theory [32] are applied in temporal query languages, including TSPARQL [23], TOQL [11], and SQWRL [37].

This section is divided into two parts. In Subsection 2.1, several well-known models of time for the semantic web including Temporal Description Logics (TDLs), OWLTime, 4D-fluentes, N-ary, Reification, and Named Graphs are discussed. Subsection 2.2 presents famous approaches based on these models which define time in their respective points of view.

\section{A. Temporal Models for RDF and $O W L$}

The Web Ontology Language (OWL) forms the basis of the semantic web and it is the most expressive standard language in modeling ontologies. However, until now, no standard approach has been introduced to express timedependent information in OWL [30].

RDF and OWL are based on binary relationships between concepts [3], [13] which simply connect two instances without any temporal information (e.g., medicine with patient). Dealing with information that changes over time or events that occur in time instants is a critical problem in the knowledge base. Nevertheless, representation of temporal information in OWL is a complex yet necessary task because the temporal dimension is central in many areas of science.

Time may be involved in concepts in two manners: implicitly and explicitly. Therefore, it is somewhat complicated to check and model the notion of time. To illustrate this, temporal models are introduced using a simple example.

The same medicine consumption time intervals for two patients are as follows:

Event1 occurs in TimeInterval1: ['2002-01-10',' 200202-10']: The drug (D1) is prescribed for patient1.

Event2 occurs in TimeInterval1: ['2002-01-10', 200202-10']: The drug (D1) is prescribed for patient2.

Event3 occurs in TimeInterval2: ['2010-09-15', 2010 11-15']: The drug (D1) is prescribed for patient1.

If this information is to be recorded in an ontology, according to the rules of OWL, we have the following relationship:

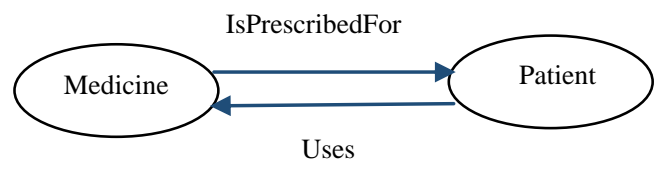

Fig.1. Concepts and objectProperty

In the following, an overview of several common temporal models is presented:

1. Temporal Description Logic (TDL) [4], [14], [20], [33]: This is an extension of classical logic [10], [12], which incorporates the concept of time into its models. The most important operators include 'since'(S), until' (U), 'always in the past' (ם), 'sometime in the future' $(\diamond)$, and 'in the next moment in future' (O). The drawback of TDL is that it cannot fully cover the Allen theorem and it is not compatible with existing OWL editing and reasoning tools.

2. OWL-Time [26]: This model is presented in the form of temporal ontologies and contains information about the temporal content of web pages and web services. It contains expressions to describe temporal relations. All temporal models presented after this model, apply basic time concepts used in this ontology. Temporal relationships in this ontology are based on time intervals.

3. 4D-Fluents [48], [7]: 4D-fluent represents temporal concepts in OWL. Time is a fourth dimension used to represent temporal concepts. The entities consist of temporal parts (time slices) that represent the entity during a time interval. Two classes i.e. TimeSlice and TimeInterval and two object properties of TimeSliceOf and tsTimeIntervalOf must be added to this model. This method increases the complexity of the ontology because for each non-temporal entity, (Patient), one individual with TimeSlice (Patient1TimeSlice1,

Patient2TimeSlice2) must be created.

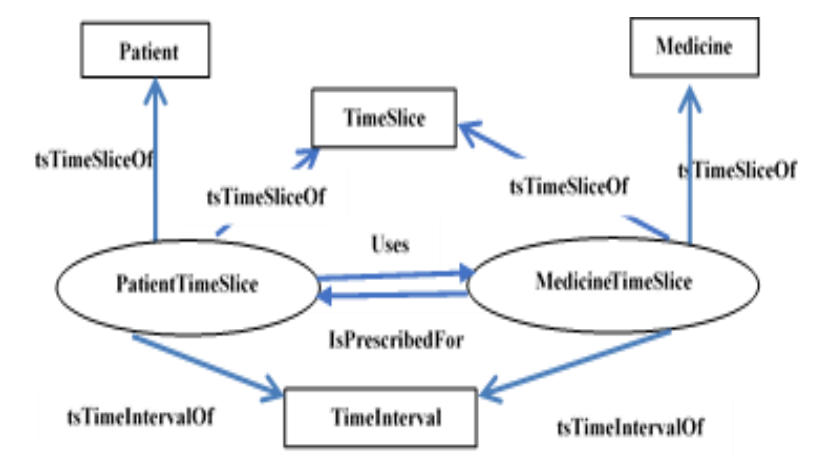

Fig.2. The 4D-Fluent model described based on the sample above

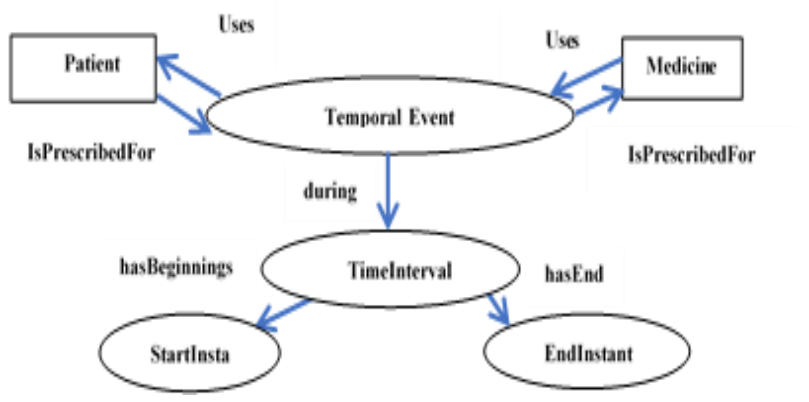

Fig.3. The $\mathrm{N}$-ary model described based on the sample above

4. The N-ary relations [36]: This approach suggests two object properties and a new object (Temporal Event) that occurs during time intervals. This approach requires only one additional object and two object properties for every temporal relation which causes minimal data redundancy in comparison with other methods. Additional properties provide binary links to each argument of the relation.

5. Reification [10] is a general-purpose technique for representing $\mathrm{N}$-ary relations, in which a relation needs to hold the subject and object at time $t$ (Patient uses medicine at time $\mathrm{t}$ ). A new object should be created for 
every relationship. A major disadvantage of Reification is that it offers limited reasoning capabilities, because relation $\mathrm{R}$ is represented as the object property.

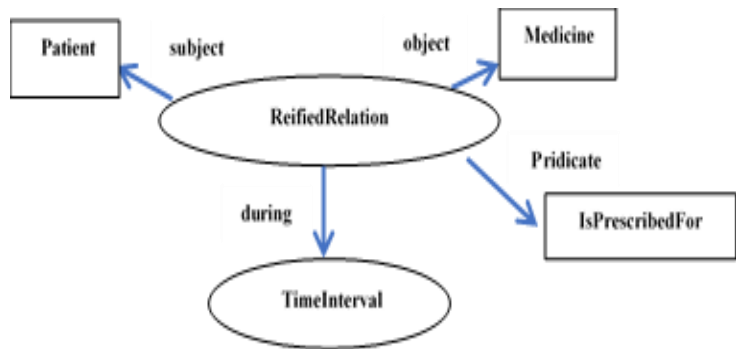

Fig.4. The Reification model described based on the sample above

6. Named Graph [47] is a sub-graph into the RDF graph of the ontology specified by a distinct name. OWL constructs are not translated into named graphs and they are not supported by OWL reasoners.

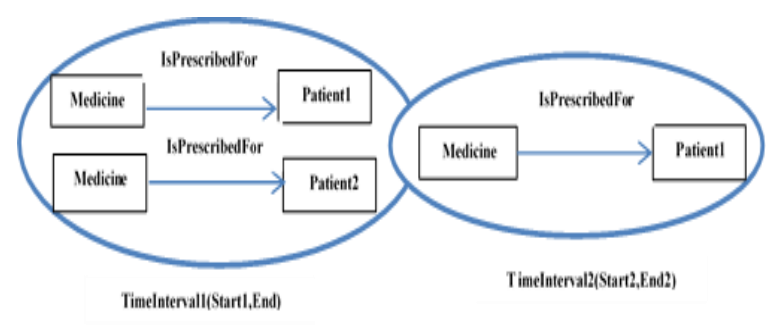

Fig.5. The Named Graph model described based on the sample above

7. The Semantic Web Rule Language (SWRL) [28], [40] is based on a combination of OWL-DL and OWL-Lite, which provides a standard method for representing temporal information in OWL ontologies. SWRL represents a mechanism for using Allen's interval rules.

Examples of systems and ontologies based on the seven described temporal models can be seen in Table 1 .

Table 1. Temporal systems and ontologies

\begin{tabular}{|c|c|}
\hline References & Description \\
\hline $\begin{array}{l}\text { [Batres et al., (2007)]; [Batsakis and Petrakis (2011)]; } \\
\text { [Zamborlini and Guizzardi (2010)]; MUSING } \\
\text { system :[Krieger (2010)] }\end{array}$ & The examples of 4D-fluents representations. \\
\hline $\begin{array}{l}\text { [Champin and Passant (2010)]; [Shaw et al. (2009)]; } \\
\text { [Wang et al. (2010)] }\end{array}$ & The examples of Reification representations. \\
\hline CNTRO [Cui et al. (2010)] & $\begin{array}{l}\text { This ontology is a temporal representation that combines SWRL rules and } \\
\text { OWL-Time for representing clinical narratives. }\end{array}$ \\
\hline VPR [O’Connor, M.J.(2008)]] & $\begin{array}{l}\text { This is a web-based application that allows users to enter patient } \\
\text { information which are first converted to and XML stream and then to } \\
\text { OWL entities. This Information is extracted through SQWRL queries. }\end{array}$ \\
\hline [Santos et al. (2011)] & This sample supports n-ary-based temporal ontologies \\
\hline [O’Connor, M.J.(2011)] & $\begin{array}{l}\text { Here a valid-time temporal model in OWL is developed and applied in } \\
\text { Reification mechanism. This system is developed for encoding temporal } \\
\text { information collected during a national clinical trials project. }\end{array}$ \\
\hline SOWL[Batsakis and Petrakis (2011)] & $\begin{array}{l}\text { This supports temporal relations through SWRL rules and 4D-fluents } \\
\text { model. }\end{array}$ \\
\hline PROTON [Papadakis et al. (2011)] & This is based on 4D-fluents and is implemented in Prolog. \\
\hline [O’Connor and Das (2011)] & $\begin{array}{l}\text { This supports the N-ary relations approach and the SWRL-Temporal } \\
\text { ontology. }\end{array}$ \\
\hline [Preventis et al. (2012)] & This is a tool for both $4 \mathrm{D}$ fluents and $\mathrm{n}$-ary relations. \\
\hline SOWL :[Anagnostopoulos et al. (2013)] & $\begin{array}{l}\text { Here a combination of system and specialized temporal reasoner are } \\
\text { presented in SOWL }\end{array}$ \\
\hline
\end{tabular}

\section{B. Impelimentation}

In the following, we present a review of the available temporal ontologies, a definition of time concepts and their relationships with other concepts.

\section{OWL-Time}

- DAML-Time [29] the basic model of time with definitions of temporal base concepts

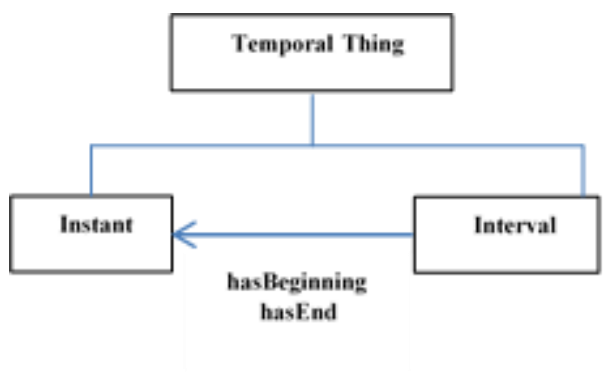

Fig.6. Temporal classes in DAML-Time 
- OWL-S [27] (formerly DAML-S) is an OWLbased Web service ontology that supplies Web service providers with a core set of markup language constructs. This is a sub-ontology of time that covers relations among instants, intervals, instant events, and interval events

2. SWRL Temporal Ontology [37] defines a temporal model for representing interval-based information in OWL. Temporal classes in this ontology include Temporal:

Granulitary, Temporal:Proposition, Temporal:Duration, and Temporal:ValidTime. A Relation in this model is based on Allen intervals, which support Positive Allen relations: durationLessThan, durationEqulTo, durationGreaterThan, equals, before, after, meets, metBy, overlaps, overlappedBy, contains, during, starts,startedBy ,finishes, finishedBy and the O'Connor relations which support negative Allen relations: notDurationLessThan, notDurationEqulTo, notDurationGreaterThan, notEquals, notBefore, notAfter, notMeets, notMetBy, notOverlaps, notOverlappedBy, notContains, notDuring, notStarts, notStartedBy, notFinishes, notFinishedBy. In addition, SWRLTemporal contains SWRL build-ins for temporal reasoning, which can be applied to check whether predicates comparing a specific duration with that of a validity interval (e.g., durationLessThan, durationEqualTo) hold true.

\section{TOWL [21]}

TOWL is a model for extending OWL-DL and 4DFluent, with the ability to reason the temporal concepts without consistency checking. Unfortunately, it cannot be compatible with the available OWL editing, querying and reasoning tools (e.g., Protégé, Pellet, and SPARQL). Most temporal models prior to TOWL had limitations in expressing temporal relations; therefore, temporal models such as $4 \mathrm{D}$-Fluent and OWL-Time were introduced to overcome these drawbacks. In TOWL, the temporal dimension is added to static concepts in a dynamic manner. The architecture of the model includes a threelayer structure, of which the first consists of static concepts with no temporal concepts. The second layer is based on fundamental temporal relationships that include Allen rules, TimeInterval and TimeInstant. Finally, the third layer consists of complex temporal concepts such as State Transition and time changes of Individual.

\section{CHORONOS [1],[41],[42]}

CHORONOS is a Tab Plugin for the Protégé editor. In this approach, the time ontology is added to the base static ontology and the user can apply the temporal ontologies as either static or dynamic. Classes and temporal relations such as Event, participatedIn (for individuals participate in event) are developed based on $\mathrm{N}$-ary model.

\section{SOWL Ontology [8]}

This ontology seeks to overcome the problem of TOWL, by offering W3C solutions for temporal representation. In SOWL models, two approaches are represented:

- The combination of OWL-time ontology and 4Dfluents model is used to represent dynamic objects. In this approach, the TimeSlice class and tsTimeSlice properties are applied.

- An alternative version based on $\mathrm{N}$-ary relations is proposed as well in [1]. Allen relations are asserted as object properties between intervals, even if exact dates are not known.

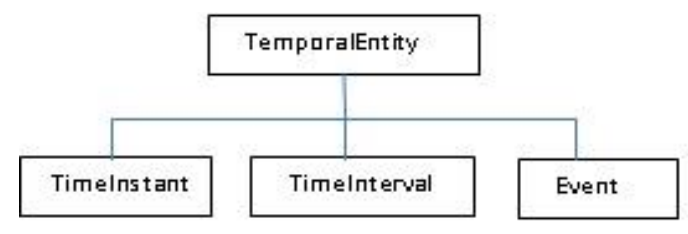

Fig.7. Temporal classes in SOWL with $\mathrm{N}$-ary

\section{CNTRO [45], [46]}

This semantic web-based framework, provides an API for querying temporal information from clinical narratives. CNTRO is an OWL ontology which contains three major components: time normalizer, SWRL, and OWL-DL reasoning. This ontology includes existing temporal ontology and Basic Formal Ontology (BFO). In this approach, temporal concepts are defined as a means for extracting information from clinical narratives. Given the fact that the Allen relations are only based on time intervals, in this ontology, an event is modeled where TimeStamp is the relation between event and time. Currently, two versions of CNTRO are available: CNTRO1 [46] and CNTRO2 [45]. The second version was released to overcome the shortcomings of the first. 


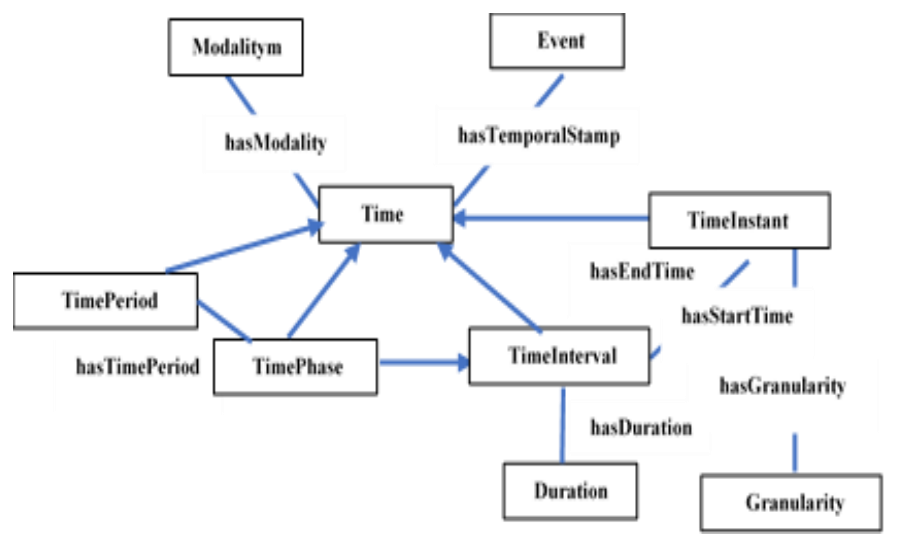

Fig.8. CNTRO concepts

7. MUSING [48] involves temporal information and investigates solutions for semantic-based business intelligence. In this approach, time slice is added to binary relations for two reasons: (I) to avoid a duplication of the original ontology and (II) to prevent a knowledge engineer from rewriting on the ontology.

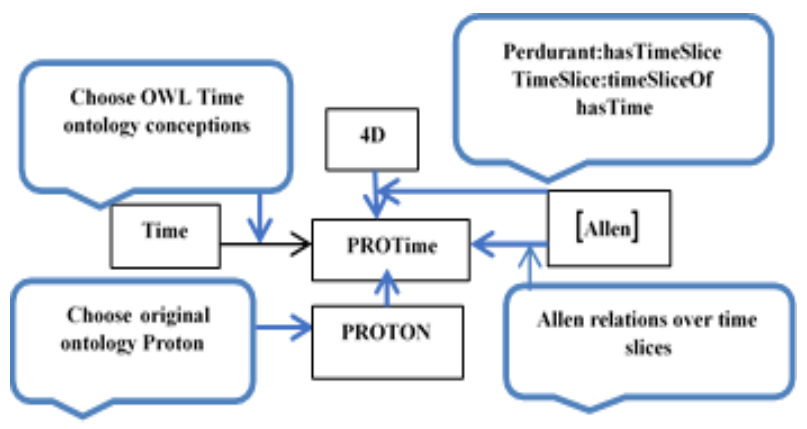

Fig.9. MUSING Components

\section{TEMPORAL QUERY LANGUAGES}

The ability to process and make inferences based on available information is the most important reason for using ontology in information integration and creating semantic relationships. Therefore, a query languages is required to enable the provision of responses using an appropriate reasoner. The main challenge is to select an appropriate query language which is able to answer most temporal and non-temporal questions. In this section, several examples of languages based on RDF and OWL are introduced. This is followed by a comparison of TOQL and SQWRL languages using two example ontologies pertaining to books by scientists in historical periods.

\section{A. XQuery Languages [16]}

Due to the important role of XML in expressing knowledge on the web and the increasing number of XML documents, it is necessary to create languages for querying and extracting knowledge from these documents. Therefore, the XQuery language was suggested under the banner of the World Wide Web Consortium. It should be noted that XQuery is developed for temporal querying of XML data.

\section{B. C-SPARQL[9]}

C-SPARQL is a language for querying over RDF data streams, which contain sequences of triples, continuously produced and annotated through timestamps. Thus, for this amount of data, stream reasoning is required.

\section{T-SPARQL[23]}

T-SPARQL is similar to TSQL2 [15]; it is a temporal extension of the SPARQL [44] for RDF. T-SPARQL is powered by the basic temporal structure designed for the TSQL2.

\section{TOQL [11]}

This is a language for querying time information in ontologies. TOQL is a SQL-like temporal query language that handles ontologies almost like relational databases. TOQL statements are similar to SQL rules, having Select, From, Where, Union etc. Time concepts in TOQL involve TimeSlice and TimeInterval. The temporal model in this language is the 4D-fluent approach. Moreover, reasoning in language is based on Event Calculus. TOQL system is implemented in Java whose user interface can be downloaded. Pellet is a reasoner in this language. The TOQL structure is composed of several modules. TOQL commands are converted to equivalent commands in SeRQL by the parser. In this language, the temporal relations of 4D-Fluent model are checked among other concepts; if all concepts as well as the temporal and nontemporal relations defined among them are correct, then the ontology is accepted and the user is allowed to run the query.

\section{E. SQWRL [38], [39]}

SQWRL is a SWRL-based query language, which defines a set of query operators similar to that of SQL that can retrieve and infer information in the OWL ontology. This language supports Allen's temporal operators. SWRL is a rule language while SQWRL is a query language. It is implemented as a build-in library and a set of query operators similar to those of SQL with the objective to extract and infer stored information in the OWL ontology. 


\section{COMPARISON BETWEEN TEMPORAL QUERY LANGUAGES}

Among the languages presented in the previous section, TOQL, SQWRL, and SOWL are more widely used for querying in ontology editors. However, since the authors were not able to access the SOWL API, the following comparison is conducted between SQWRL and TOQL using two sample ontologies .The former is implemented in the Protégé 3.5 environment and the latter in the Protégé 4.3.
TOQL is similar to SQL, whereas SQWRL is based on logic. The relationships and classes presented in Figures 10 and 11 are based on the temporal models used in these languages. As observed in the model of classes, relationships in TOQL are very complex. For every concept or class in TOQL model ontology, a relationship with TimeSlice and TimeInterval must be created. Examples of large ontologies such as CNTRO [46] are implemented using SQWRL language, while TOQL language may be appropriate for small ontologies.

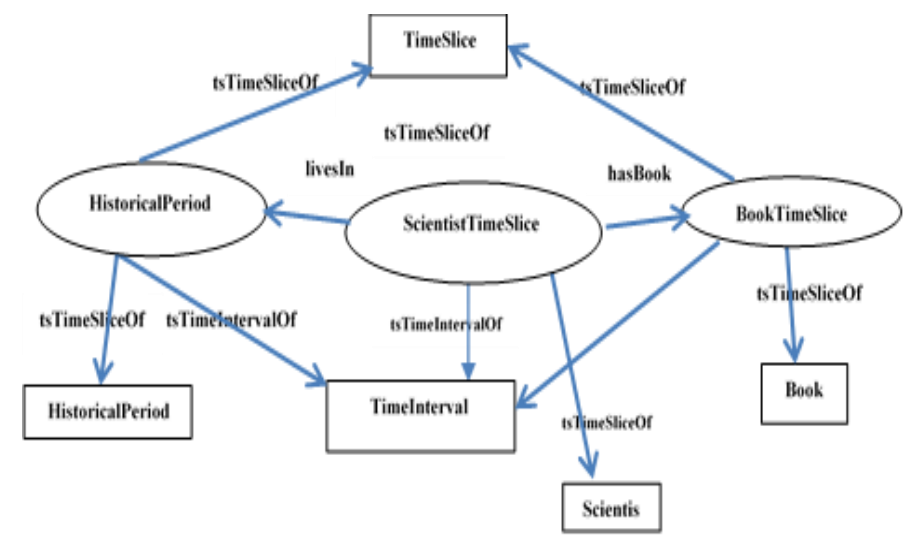

Fig.10. Classes and objectProperty in sample of TOQL

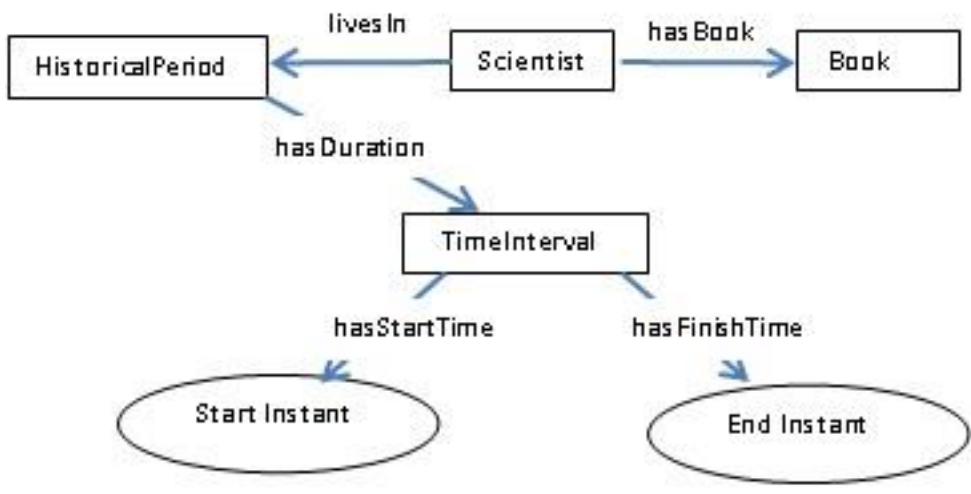

Fig.11. Classes and object Property in sample of SQWRL

The query asking for "names of scientists along with the titles of their books and time periods of their activities" is a non-temporal question, which can be presented in following languages:

\section{- SQWRL :}

Scientist $(? \mathrm{~s}) \wedge$ Books $(? \mathrm{~b}) \wedge$ hasBook $(? \mathrm{~s}, ? \mathrm{~b}) \wedge$

livesIn(?s, ?hp) $\rightarrow$ sqwrl:select(?s,?b,?hp)

\section{- TOQL :}

Select Scientist.name,

HistoricalPeriod.historicalPeriodName,Books.name

From Physician,HistoricalPeriod, Books

Where Scientist.livesIn:HistoricalPeriod and Scientist.hasBook: Book

The following temporal questions are asked in both ontology query languages, according to Allen theory.
Q1: Titles of books by scientists written in the time period $\mathrm{P}$.

Q2: Titles of books by scientists written subsequent to '800-01-06'. 01-06'

Q3: Titles of books by scientists written prior to '2007-

Q4: Titles of books by scientists written between the dates '900-01-01' and '1900-01-01'.

TOQL cannot properly respond to these questions because it is not completely capable of detecting time intervals. The following query is in TOQL.

SELECT Scientist.name, Book.name

FROM Scientist, Book

WHERE Scientist.hasBook: Book Before '2007-01$06^{\prime}$ 


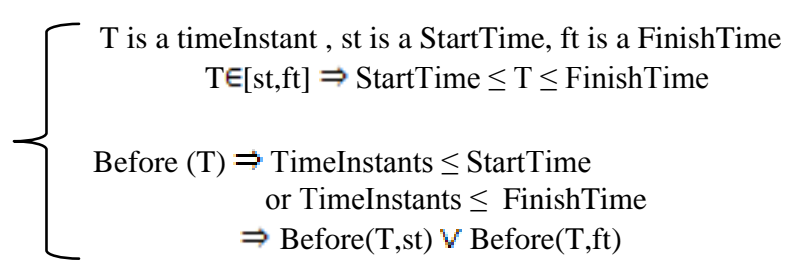

Unlike SQWRL, in TOQL, access to exact beginning, ending and during "date" is not possible.

Question Q3 is presented in two forms of Q3.1.1 and Q3.1.2 below for comparing dates before Start time and for dates before finish time as Q3.2.

Q3.1.1 : Scientist(?s) $\wedge$ temporal:ValidPeriod(?p) $\wedge$ hasDuration(?s, ?p) ^ temporal:hasStartTime $(? \mathrm{p}, ? \mathrm{start})^{\wedge}$ temporal:before(?start, “'2007-01-06’T00:00:00”, "Years") $\rightarrow$ sqwrl:select(?pe, ?p, ?start)

Q3.1.2 : Scientist(?s $)^{\wedge} \operatorname{Books}(? \mathrm{~b})^{\wedge} \operatorname{hasBook}(? \mathrm{~s}, ? \mathrm{~b})^{\wedge}$ temporal:ValidPeriod(?p) $\wedge$ hasDuration(?p, ?du) $\wedge$ hasStartYear(?du, ?s) ^ swrlb:lessThanOrEqual (?s, 2007) $\rightarrow$ sqwrl:select(?s,?b, ?s)

Q3.2 : Scientist $(\text { ?s })^{\wedge} \operatorname{Books}(? \mathrm{~b}) \wedge \operatorname{hasBook}(? \mathrm{~s}, ? \mathrm{~b}) \wedge$ temporal:ValidPeriod(?p) $\wedge$ hasDuration(?p, ?du) $\wedge$ temporal:hasFinishTime(?du, temporal:before(?finish, “'2007-01-06'T00:00:00”, "Years") $\rightarrow$ sqwrl:select(?s, ?p, ?finish)

Consequently, in TOQL, it is not possible to answer queries which compare date intervals such as OverLaps, Contains, and Meets. However, SQWRL is capable of responding to queries formed based on time intervals. Table 2 compares the specifications of the languages.

Table 2. Comparison between TOQL and SQWRL

\begin{tabular}{|c|c|c|}
\hline Properties & SQWRL & TOQL \\
\hline Complexity & Low & High \\
\hline $\begin{array}{l}\text { Query } \\
\text { Answering } \\
\text { Speed }\end{array}$ & Low & Relatively well \\
\hline $\begin{array}{l}\text { Limit the } \\
\text { number of } \\
\text { response }\end{array}$ & Unlimited & Limited \\
\hline $\begin{array}{l}\text { Creating extra } \\
\text { object }\end{array}$ & $\begin{array}{l}\text { Except SWRL classes } \\
\text { and temporal relations } \\
\text { no other relationships } \\
\text { are necessary }\end{array}$ & $\begin{array}{l}\text { For any non- } \\
\text { temporal object, } \\
\text { two additional } \\
\text { relationship are } \\
\text { required }\end{array}$ \\
\hline $\begin{array}{l}\text { Allen's interval } \\
\text { coverage }\end{array}$ & Relatively complete & Weak \\
\hline Documentation & Relatively complete & $\begin{array}{l}\text { Fairly weak sample } \\
\text { codes }\end{array}$ \\
\hline User Interface & No & Yes \\
\hline Reasoner & Pellet and Jess & Pellet \\
\hline
\end{tabular}

In TOQL, a small and limited sample of products and employees is introduced; however, for more accurate studies comprehensive samples are necessary.

The abovementioned comparison between the two languages based on 4D-Fluent and SWRL time models shows that, due to the current capabilities of OWL , the definition of temporal and non-temporal concepts in SWRL is easier and more understandable than other models and can be effective in designing ontologies as well as answering users queries.

\section{CONCLUSION}

Recognizing the defined temporal models is an important step for relating time with other concepts in any field.

In recent years, numerous applications and ontologies have been developed based on the aforementioned temporal models with the objective of incorporating time into RDF and OWL structures; each of them seeks to remove the drawbacks of its predecessors.

Another important step, after defining time, is understanding and knowing how to process the stored information based on temporal models and then selecting an appropriate language to query and extract new knowledge from the knowledge base.

In this paper, we aimed to review and partially explain the strengths and weaknesses of various models by comparing temporal models and query languages of ontologies and presenting a practical example an ontology for SQWRL and TOQL in the Protégé editor.

\section{REFERENCES}

[1] Anagnostopoulos, Eleftherios, Sotiris Batsakis, and Euripides GM Petrakis. "Chronos: A reasoning engine for qualitative temporal information in owl."Procedia Computer Science 22 (2013): 70-77.

[2] Analyti, Anastasia, and Ioannis Pachoulakis. "A Survey on Models and Query Languages for Temporally Annotated RDF." (2008).

[3] Antoniou, Grigoris, and Frank Van Harmelen. A semantic web primer. MIT press, 2004.

[4] Artale, Alessandro, et al. "A temporal description logic for reasoning over conceptual schemas and queries." Logics in Artificial Intelligence. Springer Berlin Heidelberg, 2002. 98-110.

[5] Allen, James F. "Towards a general theory of action and time." Artificial intelligence 23, no. 2 (1984): 123-154.

[6] Batsakis, Sotiris, Grigoris Antoniou, and Ilias Tachmazidis. "Integrated Representation of Temporal Intervals and Durations for the Semantic Web."New Trends in Database and Information Systems II. Springer International Publishing, 2015. 147-158.

[7] Batsakis, Sotiris, and Euripides GM Petrakis. "Representing temporal knowledge in the semantic web: The extended $4 \mathrm{~d}$ fluents approach."Combinations of Intelligent Methods and Applications. Springer Berlin Heidelberg, 2011. 55-69.

[8] Batsakis, Sotiris, and Euripides GM Petrakis. "SOWL: spatio-temporal representation, reasoning and querying over the semantic web." Proceedings of the 6th International Conference on Semantic Systems. ACM, 2010.

[9] Barbieri, Davide Francesco, Daniele Braga, Stefano Ceri, EMANUELE DELLA VALLE, and Michael Grossniklaus. "C-sparql: a continuous query language for rdf data streams." International Journal of Semantic Computing 4, 
no. 01 (2010): 3-25.

[10] Buneman, Peter, and Egor Kostylev. "Annotation algebras for RDFS." In The Second International Workshop on the role of Semantic Web in Provenance Management (SWPM-10), CEUR Workshop Proceedings, p. 32. 2010.

[11] Baratis, Evdoxios, et al. "TOQL: Temporal ontology querying language."Advances in Spatial and Temporal Databases. Springer Berlin Heidelberg, 2009. 338-354.

[12] Baader, Franz. The description logic handbook: theory, implementation, and applications. Cambridge university press, 2003.

[13] Berners-Lee, Tim, James Hendler, and Ora Lassila. "The semantic web."Scientific American 284.5 (2001): 28-37.

[14] Bettini, Claudio. "Time-dependent concepts: representation and reasoning using temporal description logics." Data \& Knowledge Engineering 22.1 (1997): 1-38

[15] Böhlen, Michael H., et al. "Querying TSQL2 databases with temporal logic."Advances in Database TechnologyEDBT'96. Springer Berlin Heidelberg, 1996. 325-341.

[16] Chamberlin, Don. "XQuery: An XML query language." IBM systems journal41.4 (2002): 597-615.

[17] Davies, John, Rudi Studer, and Paul Warren, eds. Semantic Web technologies: trends and research in ontology-based systems. John Wiley \& Sons, 2006.

[18] Embregts, Hanno, Viorel Milea, and Flavius Frasincar. "Metafrastes: A news ontology-based information querying using natural language processing." The 8th International Conference on Knowledge Management in Organizations. Springer Netherlands, 2014.

[19] Ermolayev.V.Batsakis, S.Keberle, N., Tatarintseva.O.,\&Antoniou,G. Onologies Of Time: Review And Trends. .(2014)

[20] Fisher, Michael. An Introduction to Practical Formal Methods Using Temporal Logic. John Wiley \& Sons, 2011

[21] Frasincar, Flavius, Viorel Milea, and Uzay Kaymak. tOWL: Integrating time in OWL. Springer Berlin Heidelberg, 2010.

[22] Fisher, Michael David, Dov M. Gabbay, and Lluis Vila, eds. Handbook of temporal reasoning in artificial intelligence. Vol. 1. Elsevier, 2005.

[23] Grandi, Fabio. "T-SPARQL: A TSQL2-like Temporal Query Language for RDF."ADBIS (Local Proceedings). 2010.

[24] Gutierrez, Claudio, Carlos Hurtado, and Alejandro Vaisman. "Introducing time into RDF." Knowledge and Data Engineering, IEEE Transactions on 19.2 (2007): 207218.

[25] Golbreich, Christine, and Sandrine Mercier. "Construction of the dialysis and transplantation ontology: advantages, limits, and questions about Protege OWL." Workshop on Medical Applications of Protégé, 7th International Protégé Conference, Bethesda. 2004.

[26] Hobbs, Jerry R., and Feng Pan. "Time ontology in OWL." W3C working draft27 (2006): 133. http://www.w3.org/TR/owl-time.

[27] Hobbs, J.R., Pan, F.: An ontology of time for the Semantic Web. ACM Transactions on Asian Language Processing (TALIP): Special issue on Temporal Information Processing 3(1), 66-85 (2004)

[28] Horrocks, Ian, et al. "SWRL: A semantic web rule language combining OWL and RuleML." W3C Member submission 21 (2004): 79.

[29] Hobbs, Jerry R., et al. "A daml ontology of time." online: http://www. cs. rochester. edu/_ferguson/daml/daml-time20020830. txt (2002).

[30] Krieger, Hans-Ulrich. "A General Methodology for Equipping Ontologies with Time." LREC. 2010.
[31] Kim, Sang-Kyun, et al. "Temporal ontology language for representing and reasoning interval-based temporal knowledge." The Semantic Web. Springer Berlin Heidelberg, 2008. 31-45.

[32] Kim, Jaegwon. Events as property exemplifications. Springer Netherlands, 1976.

[33] Lutz, Carsten, Frank Wolter, and Michael Zakharyaschev. "Temporal Description Logics: A Survey." TIME. 2008.

[34] Mishra, Ravi Bhushan, and Sandeep Kumar. "Semantic web reasoners and languages." Artificial Intelligence Review 35.4 (2011): 339-368.

[35] Maris, Nikos. A reasoner for querying temporal ontologies. Diss. Dept of Sciences, TEI of Crete, 2008.

[36] Noy, Natasha, Alan Rector, P. Hayes, and C. Welty. "Defining $\mathrm{N}$-ary relations on the Semantic Web. W3C working group note." World Wide Web Consortium (W3C) (2006).

[37] O'Connor, Martin J., and Amar K. Das. "A method for representing and querying temporal information in OWL." Biomedical engineering systems and technologies. Springer Berlin Heidelberg, 2011.97-110.

[38] O'Connor, Martin J., and Amar K. Das. "SQWRL: A Query Language for OWL."OWLED. Vol. 529. 2009.

[39] O'Connor, Martin J., Ravi D. Shankar, Csongor Nyulas, Samson W. Tu, and Amar K. Das. "Developing a WebBased Application using OWL and SWRL." InAAAI spring symposium: AI meets business rules and process management, pp. 93-98. 2008.

[40] O'Connor, Martin, Samson Tu, Csongor Nyulas, Amar Das, and Mark Musen. "Querying the semantic web with SWRL." In Advances in Rule Interchange and Applications, pp. 155-159. Springer Berlin Heidelberg, 2007.

[41] O'Connor, Martin J., Samson W. Tu, and Mark A. Musen. "The Chronus II temporal database mediator." In Proceedings of the AMIA Symposium, p. 567. American Medical Informatics Association, 2002.

[42] Preventis, Alexandros, Euripides GM Petrakis, and Sotirios Batsakis. "CHRONOS Ed: A tool for handling temporal ontologies in protégé."International Journal on Artificial Intelligence Tools 23, no. 04 (2014): 1460018.

[43] Papadakis, Nikos, Kostas Stravoskoufos, Evdoxios Baratis, Euripides GM Petrakis, and Dimitris Plexousakis. "Proton: A prolog reasoner for temporal ontologies in owl." Expert Systems with Applications 38, no. 12 (2011): 1466014667.

[44] Pérez, Jorge, Marcelo Arenas, and Claudio Gutierrez. "Semantics and complexity of SPARQL." ACM Transactions on Database Systems (TODS) 34, no. 3 (2009): 16.

[45] Tao, C., Solbrig, H. R., \& Chute, C. G. (2011). CNTRO 2.0: a harmonized semantic web ontology for temporal relation inferencing in clinical narratives. AMIA Summits on Translational Science Proceedings, 2011, 64.

[46] Tao, Cui, Wei-Qi Wei, Harold R. Solbrig, Guergana Savova, and Christopher G. Chute. "CNTRO: a semantic web ontology for temporal relation inferencing in clinical narratives." In AMIA Annual Symposium Proceedings, vol. 2010, p. 787. American Medical Informatics Association, 2010.

[47] Tappolet, Jonas, and Abraham Bernstein. "Applied temporal rdf: Efficient temporal querying of rdf data with sparql." In The Semantic Web: Research and Applications, pp. 308-322. Springer Berlin Heidelberg, 2009.

[48] Welty, Chris, Richard Fikes, and Selene Makarios. "A reusable ontology for fluents in OWL." In FOIS, vol. 150, pp. 226-236. 2006. 


\section{Authors' Profiles}

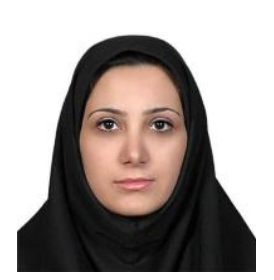

Bahadorani Bahareh: Master degree of software engineering in Azad University of Isfahan, interested in, Semantic Web, Temporal Modeling and Software Development.

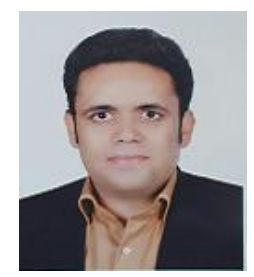

Zaeri Ahmad: Assistant professor of software engineering in university of Isfahan, interested in Semantic Web, Knowledge Mining and Software Development.

How to cite this paper: Bahareh Bahadorani, Ahmad Zaeri,"Comparison of Time Concept Modeling for Querying Temporal Information in OWL and RDF", International Journal of Information Technology and Computer Science(IJITCS), Vol.9, No.7, pp.26-34, 2017. DOI: 10.5815/ijitcs.2017.07.03 